\title{
Central Nervous System Involvement in Adults with Epidemic Hemolytic Uremic Syndrome
}

\author{
M. Wengenroth, J. Hoeltje, J. Repenthin, T.N. Meyer, F. Bonk, H. Becker, S. Faiss, O. Stammel, P.P. Urban, and R. Bruening
}

\begin{abstract}
SUMMARY: Hemolytic uremic syndrome is a multisystem disorder that is caused by infection with Shiga-toxin-producing Escherichia coli. HUS affects mainly children and is rare among adults. This retrospective case series analyzes clinical signs and MR imaging findings of 11 adult patients with HUS associated nervous system involvement during the epidemic EHEC outbreak in northern Europe with its epicenter in Hamburg in May 2011. The most prevalent imaging finding was symmetric pointy vasogenic edema of the brain stem in the acute and subacute phases of the disease $(n=5)$. One patient exhibited additional symmetric mesiotemporal signal changes mimicking limbic encephalitis. Two patients developed subcortical patchy lesions, and 4 subjects did not present with any signal changes. Remarkably, territorial ischemia, signs of hemorrhage, or blood-brain barrier disruption have not been detected. While brain stem lesions were transient and normalized with clinical recovery, supratentorial lesions did not resolve completely at 2-month follow-up examination.
\end{abstract}

ABBREVIATIONS: EHEC = enterohemorrhagic Escherichia coli; HUS = hemolytic uremic syndrome; PRES = posterior reversible encephalopathy syndrome; $\mathrm{STEC}=$ Shiga toxin-producing E coli

$\mathrm{H}$ emolytic uremic syndrome is a multisystem disorder that is triggered by bacterial gastroenteritis with Shiga toxin-producing enterohemorrhagic Escherichia coli. Typically HUS sporadically affects children younger than 3 years of age and is characterized by acute renal failure, thrombocytopenia, and microangiopathic hemolytic anemia. ${ }^{1}$ Even though central nervous system involvement is a known and frequent complication in infants with HUS $\left(20 \%-50 \%\right.$ of cases $\left.^{2,3}\right)$, the scarce body of literature mainly consists of case reports. ${ }^{2-12}$ Only a very few retrospective studies on larger pediatric patient groups with HUSassociated encephalopathy exist. ${ }^{13-15}$ The pathophysiology of neurologic complications still remains enigmatic; therefore, the treatment is essentially empiric. ${ }^{16}$

Neuroimaging studies in children revealed mainly bilateral

Received April 26, 2012; accepted after revision July 20.

From the Departments of Radiology (M.W., J.H., F.B., R.B.), Neurology (J.R., O.S., P.P.U.), Nephrology (T.N.M.), Pulmonology and Intensive Care (H.B.), and Gastroenterology (S.F.), Asklepios Clinic Barmbek, Hamburg, Germany; and Department of Neuroradiology (M.W.), Heidelberg Medical School, Heidelberg, Germany.

J.R. and T.N.M. participated in a pharmacologic study for HUS treatment (STEC-

HUS study), which was funded by Alexion. The funders had no role in study design, data collection and analysis, decision to publish, or preparation of the manuscript for this study. The authors state no other conflict of interest.

Please address correspondence to M. Wengenroth, MD, or R. Bruening, MD, Asklepios Clinic Barmbek, Rübenkamp 220, 22291 Hamburg, Germany; e-mail: martina.wengenroth@med.uni-muenchen.de or r.bruening@asklepios.com

- Indicates open access to non-subscribers at www.ajnr.org

三 Indicates article with supplemental on-line table

http://dx.doi.org/10.3174/ajnr.A3336

symmetric lesions of the basal ganglia ${ }^{4,6,15}$ or extensive cortical and/or subcortical lesions, ${ }^{14}$ which may or may not be reversible.

In contrast to the clinical presentation in children, HUS is generally a rare condition in adults, and it is not commonly associated with neurologic symptoms. To our knowledge, only 2 cases of young adults in their early 20s with HUS-associated encephalopathy have been reported in the literature so far. ${ }^{17,18}$

Given the generally rare incidence of HUS in adults, the recent epidemic with its epicenter in Hamburg and northern Germany, which occurred in May and June 2011, was all the more puzzling, because first, mainly immunocompetent adults were affected ( $89 \%$ were older than 17 years of age; median age, 43 years), and second, they often developed neurologic complications. ${ }^{19}$ As of July 18, 2011, 3222 EHEC outbreak cases (including 39 deaths) had been reported in Germany, including 852 with HUS and 32 HUS-associated deaths. ${ }^{19}$ The cause of this epidemic has been identified as an unusually aggressive enterohemorrhagic $E$ coli (EHEC) type O104:H4 ST 678, most likely transferred by contaminated raw bean sprouts. ${ }^{19-21}$ These Shiga toxin 2- and extended spectrum $\beta$ lactamase-producing bacteria are known to be resistant to most antibiotic treatments.

\section{MATERIALS AND METHODS Case Series}

Within the course of this recent EHEC epidemic, 68 patients with confirmed EHEC infection were treated in the Asklepios Clinic Barmbek in Hamburg (47 women, 69\%; 21 men, 31\%; mean age, 


\begin{tabular}{|c|c|c|c|c|}
\hline & $\begin{array}{c}\text { Peracute } \\
\text { (Day 0) }\end{array}$ & $\begin{array}{c}\text { Acute } \\
\text { (Days 1-5) }\end{array}$ & $\begin{array}{c}\text { Subacute } \\
\text { (Days 10-28) }\end{array}$ & $\begin{array}{l}\text { Late Follow-Up } \\
\text { (Days 41-102) }\end{array}$ \\
\hline Vasogenic edema of central pons & $0 / 2$ & $5 / 9$ & $1 / 5$ & $0 / 11$ \\
\hline Symmetric patchy subcortical/cortical hyperintensities & $0 / 2$ & $3 / 9$ & $3 / 5$ & $4 / 11$ \\
\hline Symmetric patchy or laminar hyperintensities of basal ganglia & $0 / 2$ & $4 / 9$ & $2 / 5$ & $2 / 11$ \\
\hline Mesiotemporal signal changes & $0 / 2$ & $1 / 9$ & $1 / 5$ & $1 / 11$ \\
\hline
\end{tabular}

${ }^{a}$ MRI presentation of HUS encephalopathy at different stages of the disease (days of MR imaging performance after the onset of neurologic symptoms) presented in relation to all patients imaged at this phase of disease. Note that not all patients underwent imaging at 4 different time points.

49.7 years). The presence of EHEC infection was confirmed by polymerase chain reaction analysis of stool samples (EHEC-positive: $n=57)$; additionally Shiga toxin $2(n=33 ; 58 \%)$, Intimin gene $(n=31 ; 54 \%)$, and Shiga toxin $1(n=2 ; 4 \%)$ were detected by enzyme-linked immunosorbent assay. ${ }^{22}$

Of 68 patients with EHEC, 32 developed HUS in the course of the disease (mean age, 44.6 years; 26 women, $81.3 \%$ ). A group of 18 patients with HUS (56\%) had neuropsychiatric symptoms ranging from mild headache to massive fear, paranoia, aphasia, severe generalized seizures, delirium, and coma (17 women, 1 man; mean age, 44 years; range, $19-66$ years).

In our cohort, the detection of HUS and the onset of diarrhea preceded the development of neurologic symptoms on average by 3 and 8 days, respectively. None of the subjects developed neurologic symptoms before the HUS syndrome manifested, and there was no history of neuropsychiatric disorders (hence, no preclinical cranial MR imaging had been performed previously).

One woman (66 years of age) died immediately after the onset of neurologic symptoms (coma) due to extensive intracranial bleeding because of a hypertensive crisis and combined thrombocytopenia. The bleeding was detected by CT.

Patients with aphasia, delirium, or more severe neurologic complications underwent routine clinical MR imaging (1.5T Achieva; Philips Healthcare, Best, Netherlands) in the acute phase of neurologic symptoms $(n=9)$. Initial MR imaging in the acute phase of the disease was performed, on average, with a delay of 2.7 days following the onset of neurologic symptoms. Two patients required anesthesia for the initial MR imaging (subjects 1 and 10 in the On-line Table).

Follow-up examinations $(n=11$; two additional patients without initial MR imaging underwent neuroimaging in the subacute phase and/or after 2 months, subjects 6 and 11; On-line Table) were performed at different times, depending on the clinical presentations (On-line Table). In total, 7 patients received gadolinium in the acute phase of the disease (footnote $a$ in the On-line Table). All patients (or their next of kin, respectively) gave written informed consent. The study was approved by the local ethics board.

\section{Clinical Presentation}

All patients with HUS-associated encephalopathy included in this study were female immunocompetent adults $(n=11$; median age, 45 years; range, 29-62 years; see the On-line Table for more details). They all developed hyperexcitability syndrome with increased muscle reflexes. Some patients also had myoclonus of the extremities. Most striking, however, were neuropsychological abnormalities in all patients with encephalopathy, including massive anxiety attacks, severe memory deficits, and impaired con- centration and orientation. Two patients developed additional generalized epileptic seizures (On-line Table). Subject 1 was most severely affected and presented with symptoms that resembled limbic encephalitis, which is typically characterized by personality changes, irritability, depression, seizures, memory loss, and sometimes dementia. ${ }^{23}$

Patients with limbic encephalitis or other autoimmune encephalopathies often express increased titers of onconeural antibodies. $^{24,25}$ Therefore, onconeural antibodies were tested in blood samples of 8 patients. However, only in 1 patient were low immunoglobulin $\mathrm{M}$ antibody titers against $\mathrm{N}$-methyl-d-aspartate receptors found (subject 10 in the On-line Table). Notably, subject 1 with clinical and neuroimaging signs of limbic encephalitis (Fig 1) was negative for onconeural antibodies.

The interdisciplinary treatment approach included hemodialysis, plasmapheresis, corticosteroids, erythrocyte transfusion, and, in a few cases, the antibody eculizumab (Soliris). After 2 months, 8 patients presented with complete neurologic recovery or without measurable symptoms. All of those 8 recovered patients went back to their former jobs; however, some reported subjective lower resilience. Two other patients (subjects 4 and 6 in the On-line Table) were not able to perform in their prior work responsibilities due to mild depression and/or reduced resilience. At the time of this article preparation, subject 1 was fully awake but still had cognitive deficits, confusion, and relapsing seizures. Because neither comorbidities nor any history of pre-existing neuropsychiatric symptoms was present, it remains uncertain why this patient had such severe symptoms in contrast to other patients of our cohort.

\section{Neuroimaging}

Unexpectedly, none of the patients with HUS investigated in our study showed signs of acute or subacute microbleeds on susceptibility-weighted or T1-weighted images. Furthermore, none had any territorial infarctions.

The overall severe clinical presentation did not correlate with extensive lesion load in neuroimaging. The most characteristic MR imaging findings of the acute phase were marked symmetrical pointy T2 hyperintensities in the dorsal pontine base $(n=5$; see the Table, On-line Table, and Fig 2). In addition to such central pontine signal alterations, 1 patient presented with bilateral T2 hyperintensities in the thalamus and mesiotemporal lobe, mimicking severe limbic encephalitis (Fig 1). Others developed juxtacortical and/or periventricular white matter ( $n=3$; Table).

Furthermore, 4 patients presented with bilateral or unilateral signal alterations of the dorsolateral thalamus (pulvinar), partially extending to the posterior limb of the internal capsule. The lentiform nucleus was not affected in this group. 


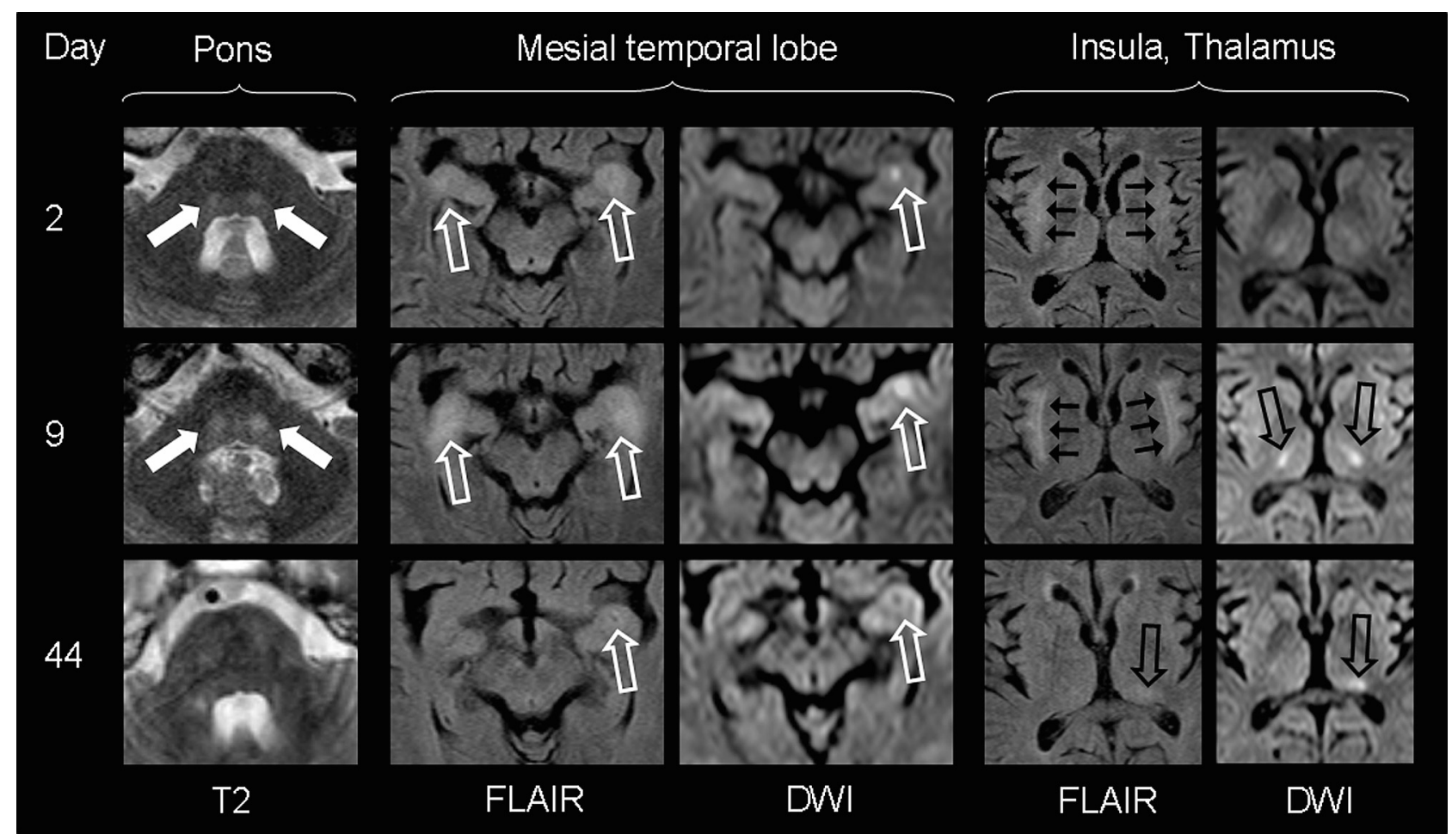

FIG 1. MR imaging findings of subject 1, presenting with delirium and severe generalized seizures. Transient bilateral laminar signal alterations of the insula (short black arrows), amygdala, and ventral hippocampus (open white arrows) mimicking limbic encephalitis are most prominent 9 days after the onset of neurologic symptoms. Marked DWI signal increase in the bilateral thalamus (open black arrows) corresponds with unaltered ADC values (not shown), indicative of vasogenic edema. Signal alterations shift from the bilateral mediolateral thalamus to the left posterolateral thalamus (pulvinar) with time. Note transient patchy diffusion restriction with low ADC values (not shown) in the left amygdala and hippocampal complex, suggestive of cytotoxic edema (either as a result from status epilepticus or as a neurotoxic and/or inflammatory effect). Additionally, bilateral round signal hyperintensities (filled white arrows) can be visualized in the central pons, normalizing with clinical improvement.

In the acute phase of the disease, 5 patients were administered intravenous gadolinium as a contrast material. Sixteen postcontrast scans were obtained in all patients with time. None of the investigated patients showed signs of a blood-brain barrier disruption or meningeal enhancement.

In follow-up examinations, pointy signal changes of the pons disappeared in all patients, correlating with clinical improvement. Even in subject 1 , who presented with severe infra- and supratentorial lesions in the acute phase of the disease, pontine signal hyperintensities were completely transient, whereas supratentorial cerebral signal changes persisted (Fig 1).

The sensitivity for signal alterations was highest in T2weighted and FLAIR sequences; however, pontine signal changes were more reliably detected in T2WI compared with FLAIR imaging (eg, Fig 2).

Diffusion-weighted imaging, including ADC, often had normal findings, even in the presence of acute, extensive lesions on T2WI (Fig 3). In 4 patients, however, focal areas of increased DWI signal were present in the acute and subacute clinical states. Brain stem lesions with increased DWI signals correlated especially with increased signal on the ADC map, suggestive of vasogenic edema. Such alterations were always transient and disappeared without any residual signal changes (Fig 3). In contrast, the patchy DWI restriction in the left amygdala and hippocampal complex of subject 1 was interpreted as suggestive of cytotoxic edema (ie, decreased ADC signal) and left a small residual scar on follow-up examinations (Fig 1).

\section{DISCUSSION}

In the pediatric population, central nervous system involvement is a known complication of hemolytic uremic syndrome. In contrast, HUS-associated encephalopathy has been largely unknown in adults. This feature might be due to the fact that HUS is generally a rare disease in adults and previous outbreaks were caused by other EHEC strains not affecting the nervous system in such an aggressive way. The pathophysiology of HUS encephalopathy is still not understood, ${ }^{26,27}$ and whether it shares a common etiology in pediatric and adult populations currently remains an open question.

Regarding previous reports of pediatric HUS encephalopathy cases and small patient groups, the main HUS-associated lesion patterns in children can be summarized as follows:

- The neurologic outcome is usually favorable despite possible residual brain lesions. Some authors report complete clinical and neuroimaging recovery, but so far no reliable predictive parameter for clinical outcome could be established. ${ }^{15}$ Hemorrhagic components within acute lesions might be indicative of residual brain lesions at later stages and poorer clinical outcome. $^{2,28}$

- Marked areas of increased DWI signal (mostly vasogenic edema) are the most common neuroimaging finding. In a number of cases, accompanying hemorrhagic components have been reported. ${ }^{2}$ To the best of our knowledge, there have not been reports of territorial brain infarctions. 


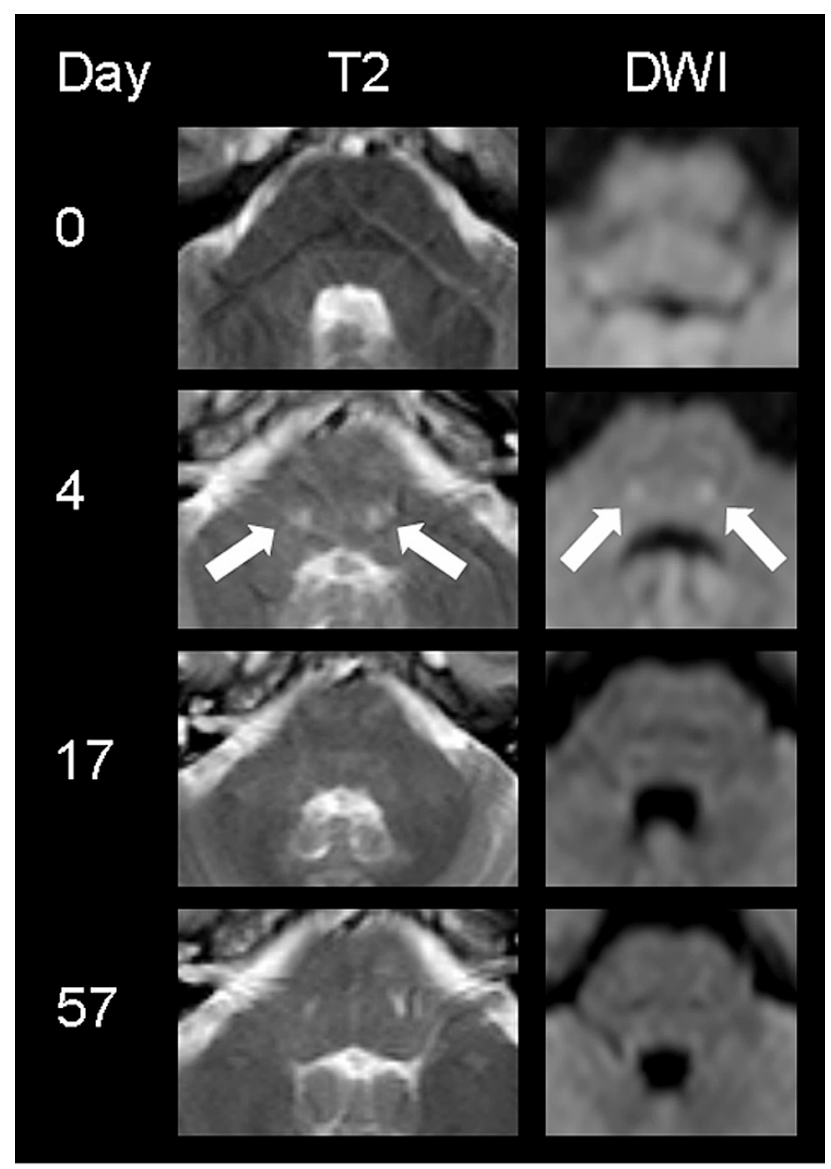

FIG 2. MR imaging findings of subject 2 presenting with aphasia, apraxia, and memory deficits. At the level of the fourth ventricle, symmetric round signal hyperintensities (filled white arrows) can be visualized in the central pons, normalizing with clinical improvement at day 17. (The DWI image on day 4 has been modified from Reference 22.)

- Lesions are often symmetric or nearly symmetric, ${ }^{2,3}$ less frequently unilateral. ${ }^{16}$

- The main target sites are the basal ganglia, including surrounding white matter structures, ${ }^{2,3,5,8}$ thalamus, and/or occipitoparietal white and gray matter structures mimicking posterior reversible encephalopathy syndrome. $9,11,17,18,28$

- A very few authors also reported additional symmetric signal alterations of the brain stem. ${ }^{2,8,14,17}$

If one compares neuroimaging findings of the patients presented in this study with those in the literature, there seem to be similarities but also marked differences between HUS-associated encephalopathy in children and adults:

- Severe neurologic symptoms do not always correlate with extensive lesion load in neuroimaging in infants and adults alike. The neurologic outcome is generally good in both groups.

- Symmetric bilateral lesion distribution is a known feature in the infant population, characteristic in our adult group as well. This is supportive of the hypothesis that the underlying pathomechanism might be of neurotoxic and/or inflammatory origin (rather than ischemic or hemorrhagic; ie, as a response to the Shiga toxin).

- Much to our surprise, none of the patients in this study demonstrated hemorrhagic components or gadoliniumenhancing lesions. The latter might be partially explained by the fact that all the patients received steroids as part of their treatment strategy, which, in turn, might have masked potential blood-brain barrier disruption. However, the fact that accompanying microbleeds are a common finding in infants but were completely absent in our adult population might be indicative of certain differences in the underlying pathology and/or capillary resilience between the groups.

- The basal ganglia were less frequently and less extensively affected in adults compared with children. Furthermore, none of the patients presented with occipital symmetric signal changes typical for posterior reversible encephalopathy syndrome.

- Marked areas of acute vasogenic edematous changes (but no territorial infarctions) are typical neuroimaging findings in children and adults alike.

- Reversible symmetric vasogenic edema of the central pons was the most common imaging finding in adults, which has been rarely described in infants. ${ }^{2}$

The limitations of the study were mainly its retrospective nature with a relatively small sample size, which did not allow meaningful statistics. However, a number of careful conclusions regarding potential pathomechanisms and imaging parameters for outcome can be drawn from the descriptive analysis of imaging findings in this rare clinical case series of adult HUS-associated encephalopathy.

In light of the recent article of Magnus et al, ${ }^{29}$ who reported perivascular gliosis predominantly found in the thalamus and pons in the postmortem brains of 5 patients with HUS-associated encephalopathy (from the Hamburg 2011 cohort), pathologic and neuroimaging findings point to a toxin-induced neuronal edema and/or autoimmune-mediated encephalopathy.

Because there are neither specific nor sensitive imaging signs, HUS encephalopathy is still a clinical diagnosis, which can only be corroborated by MR imaging. However, transient symmetric vasogenic edema of the central pons in adult patients with HUS with neurologic complications is the most common finding and therefore highly indicative. Previously, reversible mild $\mathrm{T} 2$ hyperintensities of the brain stem have been observed in different kinds of encephalopathy (ie, due to drug exposure such as to metronidazole ${ }^{30}$ or due to virus infection such as herpes simplex. ${ }^{31}$ ). In some patients, potential lesions might have been overlooked due to the fact that pontine hyperintensities measured $4-7 \mathrm{~mm}$ in diameter and all sequences applied within this clinical routine imaging protocol had a section thickness of $5 \mathrm{~mm}$. At this stage, it was not possible to restrict the lesions to exclusive brain stem nuclei because they were localized in a similar but not completely identical position in the dorsocentral pons. In this context, none of the patients with such pontine signal alterations or any other patients with HUS demonstrated isolated symptoms of brain stem lesions such as oculomotor disturbances.

Most interesting, symmetric pontine signal changes were transient in all 5/11 subjects and correlated with good clinical outcome. On the other hand, patchy and laminar supratentorial lesions alike were not completely reversible and could still be detected at 2-month follow-up. This pattern also held up in 


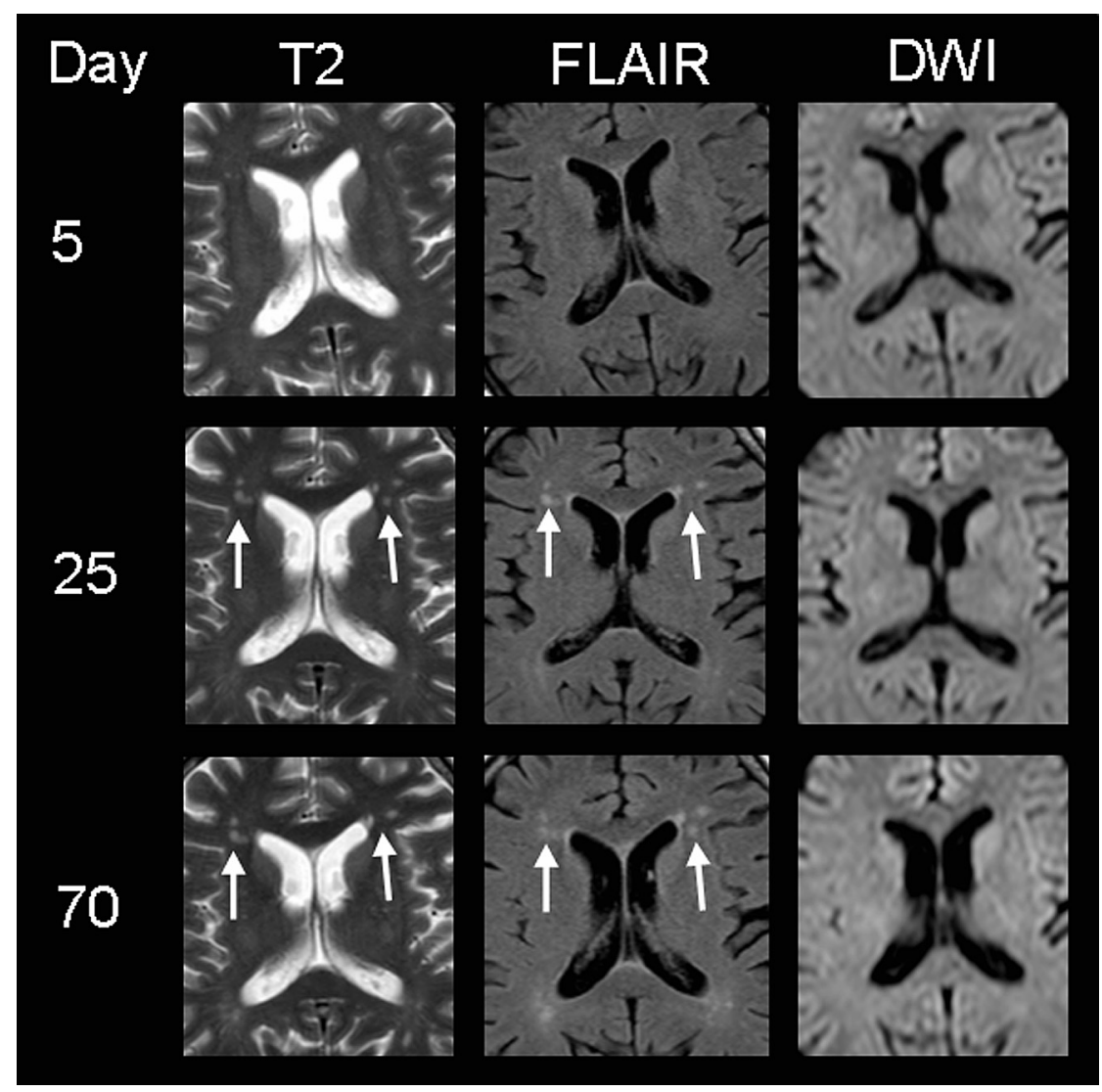

FIG 3. MR imaging findings of subject 3 , presenting with delirium and memory deficits. Note the patchy hyperintensities on T2WI and FLAIR images in the frontal periventricular white matter (white arrows) 3 weeks after the onset of neurologic symptoms (supposedly as a result of HUS encephalopathy because they could not be detected on day 5). DWI shows no restriction, indicating that no ischemia is present. At this stage, the patient did not show any neurologic symptoms. After 10 weeks, these lesions are still visible despite complete clinical recovery. siotemporal signal changes had the worst outcome with residual seizures after 2 months. Because in our cohort no lesions were detected on the day neurologic symptoms occurred, performing MR imaging at least 1 day after symptom onset is recommended. Whether there is a correlation with residual lesion load and clinical outcome remains to be investigated in later follow-up examinations and possibly in a larger group.

\section{ACKNOWLEDGMENTS}

We thank Karl Wagner (Department of Nephrology) and Thies Fitting (Department of Radiology) for their contributions in patient care and imaging interpretation, respectively.

Disclosures: Jonas Repenthin-UNRELATED: Employment: Asklepios Hamburg GmbH, Payment for Lectures (including service on Speakers Bureaus): workshop, Comments: Desitin GmbH (April 21, 2012), Biogen Idec (November 5, 2011), Payment for Development of Educational Presentations: Asklepios Medical School, Other: Roche (Opera), ${ }^{*}$ Genzyme (Care MS),* Novartis (Fingolimod),* Comments: study investigator. Oliver StammelUNRELATED: Asklepios Klinik Barmbek, Payment for Lectures (including service on Speakers Bureaus): Fa. Abbot $\mathrm{GmbH}$, Comments: about Parkinson disease. Roland Bruening-UNRELATED: Pay ment for Lectures (including service on Speakers Bureaus): lecture activity for Bracco (Germany) and GE Healthcare (Germany). *Money paid to the institution. subject 1 , who presented with combined brain stem and cerebral lesions; at the time of the article preparation, infratentorial lesions were normalized and supratentorial lesions were reduced but still visible. It remains to be seen in future repeat MR imaging if supratentorial lesions might disappear at later stages.

As summarized in the Table, when MR imaging has been performed on the very same day as symptom onset, no signal changes could be observed in our group. If this observation can be corroborated in a larger patient group, performing MR imaging would be recommended at least 1 day after the onset of neurologic complications in patients with HUS.

\section{CONCLUSIONS}

The aim of this study was to identify predictive markers for good and poor clinical outcomes in HUS encephalopathy in adults, to assist clinicians and radiologists in the interpretation of clinical and neuroimaging signs in this population. In summary, nearly all patients with HUS with neurologic complications had a generally good clinical outcome. Many patients did not present with any lesions on cranial MR imaging. The most prevalent imaging marker was transient symmetric vasogenic edema of the central brain stem. Patients with additional or solely supratentorial lesions also had a satisfactory outcome; however, these lesions were still detectable on 2-month follow-up scans. The patient with me-

\section{REFERENCES}

1. Griffin PM, Ostroff SM, Tauxe RV, et al. Illnesses associated with Escherichia coli O157:H7 infections: a broad clinical spectrum. Ann Intern Med 1988;109:705-12

2. Toldo I, Manara R, Cogo P, et al. Diffusion-weighted imaging findings in hemolytic uremic syndrome with central nervous system involvement. J Child Neurol 2009;24:247-50

3. Sherwood JW, Wagle WA. Hemolytic uremic syndrome: MR findings of CNS complications. AJNR Am J Neuroradiol 1991;12:703-04

4. Jeong YK, Kim IO, Kim WS, et al. Hemolytic uremic syndrome: MR findings of CNS complications. Pediatr Radiol 1994;24:585-86

5. Ogura H, Takaoka M, Kishi M, et al. Reversible MR findings of hemolytic uremic syndrome with mild encephalopathy. AJNR Am J Neuroradiol 1998;19:1144-45

6. Hager A, Staudt M, Klare B, et al. Hemolytic-uremic syndrome with involvement of basal ganglia and cerebellum. Neuropediatrics 1999;30:210-13

7. Yasuhara A, Araki A, Ochi A, et al. Magnetic resonance imaging of brain lesions of a patient with hemolytic uremic syndrome following Escherichia coli O157 infection. Pediatr Int 2000;42:302-05

8. Signorini E, Lucchi S, Mastrangelo M, et al. Central nervous system involvement in a child with hemolytic uremic syndrome. Pediatr Nephrol 2000;14:990-92

9. Schmidt S, Gudinchet F, Meagher-Villemure K, et al. Brain involvement in haemolytic-uraemic syndrome: MRI features of coagulative necrosis. Neuroradiology 2001;43:581-85

10. Bennett B, Booth T, Quan A. Late onset seizures, hemiparesis and blindness in hemolytic uremic syndrome. Clin Nephrol 2003;59:196-200 
11. Vergouwen MD, Adriani KS, Roos YB, et al. Proximal cerebral artery stenosis in a patient with hemolytic uremic syndrome. AJNR Am J Neuroradiol 2008;29:e34

12. Koehl B, Boyer O, Biebuyck-Gouge N, et al. Neurological involvement in a child with atypical hemolytic uremic syndrome. Pediatr Nephrol 2010;25:2539-42

13. Theobald I, Kuwertz-Broking E, Schiborr M, et al. Central nervous system involvement in hemolytic uremic syndrome (HUS): a retrospective analysis of cerebral CT and MRI studies. Clin Nephrol 2001;56:S3-8

14. Steinborn M, Leiz S, Rudisser K, et al. CT and MRI in haemolytic uraemic syndrome with central nervous system involvement: distribution of lesions and prognostic value of imaging findings. Pediatr Radiol 2004;34:805-10

15. Nathanson S, Kwon T, Elmaleh M, et al. Acute neurological involvement in diarrhea-associated hemolytic uremic syndrome. Clin J Am Soc Nephrol 2010;5:1218-28

16. Lapeyraque AL, Malina M, Fremeaux-Bacchi V, et al. Eculizumab in severe Shiga-toxin-associated HUS. N Engl J Med 2011;364:2561-63

17. Nakamura $H$, Takaba $H$, Inoue $T$, et al. MRI findings of hemolytic uremic syndrome with encephalopathy: widespread symmetrical distribution. J Neuroimaging 2003;13:75-78

18. Taylor MB, Jackson A, Weller JM. Dynamic susceptibility contrast enhanced MRI in reversible posterior leukoencephalopathy syndrome associated with haemolytic uraemic syndrome. $\mathrm{Br} J$ Radiol 2000;73:438-42

19. Frank C, Werber D, Cramer JP, et al. Epidemic profile of Shigatoxin-producing Escherichia coli O104:H4 outbreak in Germany. N Engl J Med 2011;365:1771-80

20. Bielaszewska M, Mellmann A, Zhang W, et al. Characterisation of the Escherichia coli strain associated with an outbreak of haemolytic uraemic syndrome in Germany, 2011: a microbiological study. Lancet Infect Dis 2011;11:671-76
21. Buchholz U, Bernard H, Werber D, et al. German outbreak of Escherichia coli O104:H4 associated with sprouts. $N$ Engl J Med 2011;365:1763-70

22. Dücker C, Dautel P, Wagner K, et al. Clinical symptoms, treatment and outcome of EHEC and EHEC-HUS patients treated as in-patients [in German]. Dtsch Med Wochenschr 2011;136:1770-76

23. Gultekin SH, Rosenfeld MR, Voltz R, et al. Paraneoplastic limbic encephalitis: neurological symptoms, immunological findings and tumour association in $\mathbf{5 0}$ patients. Brain 2000;123(pt 7):1481-94

24. Vincent A, Bien CG, Irani SR, et al. Autoantibodies associated with diseases of the CNS: new developments and future challenges. Lancet Neurol 2011;10:759-72

25. Flanagan EP, Caselli RJ. Autoimmune encephalopathy. Semin Neurol 2011;31:144-57

26. Boccoli J, Loidl CF, Lopez-Costa JJ, et al. Intracerebroventricular administration of Shiga toxin type 2 altered the expression levels of neuronal nitric oxide synthase and glial fibrillary acidic protein in rat brains. Brain Res 2008;1230:320-33

27. Fujii J, Kinoshita Y, Matsukawa A, et al. Successful steroid pulse therapy for brain lesion caused by Shiga toxin 2 in rabbits. Microb Pathog 2009;46:179-84

28. Fujii K, Matsuo K, Takatani T, et al. Multiple cavitations in posterior reversible leukoencephalopathy syndrome associated with hemolytic-uremic syndrome. Brain Dev 2012;34:318-21

29. Magnus T, Röther J, Simova O, et al. The neurological syndrome in adults during the 2011 northern German $E$. coli serotype O104:H4 outbreak. Brain 2012;135:1850-59

30. Miura S, Kurita T, Noda K, et al. Symmetrical brainstem encephalitis caused by herpes simplex virus. J Clin Neurosci 2009;16:589-90

31. Kim E, Na DG, Kim EY, et al. MR imaging of metronidazole-induced encephalopathy: lesion distribution and diffusion-weighted imaging findings. AJNR Am J Neuroradiol 2007;28:1652-58 